\title{
The One Hand Wonder \\ A Framework for Enhancing One-handed Website Operation on Touchscreen Smartphones
}

\author{
Karsten Seipp and Kate Devlin \\ Department of Computing, Goldsmiths College, University of London, Lewisham Way, SE14 6NW, London, U.K.
}

Keywords: One-handed Operation, Mobile, Web, Wheel Menu, Interface Adaptation, CSS3, JavaScript, HTML.

\begin{abstract}
Operating a website with one hand on a touchscreen mobile phone remains a challenging task: solutions to adapt websites for mobile users do not address the ergonomic peculiarities of one-handed operation. We present the design and evaluation of the One Hand Wonder (OHW) - an easily-adaptable cross-platform JavaScript framework to support one-handed website navigation on touchscreen smartphones. It enhances usability without the need to redesign the existing website or to overwrite any CSS styles. User testing and quantitative evaluation confirm learnability and efficiency with clear advantages over non-enhanced browsing, and a discussion of the OHW's versatility is given.
\end{abstract}

\section{INTRODUCTION}

The majority of smartphones sold today use modern operating systems such as Android and iOS, both of which have a powerful and largely standardcompliant browser paired with a touchscreen interface. Numerous websites already automatically adapt their layout and handling to the constraints of the access device to provide an adequate user experience, using web technologies such as JavaScript, CSS3 media queries or server-sided device detection.

With the help of established adaptation techniques for websites on mobile devices (W3C, 2008; ASA, 2007) as well as responsive themes (Envato, 2013), device-independent websites are becoming the norm. In addition, further approaches exist to adapt websites to mobile device constraints, although these are either proprietary (Akmin, 2012), dependent on a proxy server (Gupta et al., 2007) or bound to a specific browser (Mobotap, 2012; Yu and Miller, 2011). These result in an adapted and improved display on a range of mobile devices, but not in an adapted interaction model for thumb-based use.

For non-adapted pages, built-in actions such as pinching and tapping to zoom can improve matters. However, none of these account for the one-handed operation of the phone, which has been identified as a preferred mode of operation by many users (Karlson and Bederson, 2006). The limited mobility and reach of the thumb represent completely different challenges to the designer regarding the layout and operation of the website; simply crafting it to ensure a correct display of the page elements does not suffice.

While some browsers (Mobotap, 2012) offer improvements for one-handed operation as part of their interface (using simple gestures, for example), we explore whether one-handed operation can be reliably improved regardless of the browser or plugin used. We do this by improving the display and control of elements operated via direct touch, without the need for gestures. As devices and browsers become increasingly powerful, the question arises as to whether such improvements can be made directly at runtime in the browser, and how efficient and usable these improvements are in comparison to non-enhanced websites.

In this paper we present a JavaScript-based framework which we have named the One Hand Wonder $(\mathrm{OHW})$. The OHW prototype provides an on-demand thumb-based interaction model for all interactive elements on a web page, facilitating operation and navigation across a wide range of websites. It gives quick access to the most common functions and elements and augments the interaction model of the browser and the standard HTML elements of a web page with additional one-handed UI features that can easily be toggled on and off. These augmentations are temporary and do not change the design of the website. The OHW is built using solely client-side technologies and is implemented by simply embedding the 
code into the the web page. Initial user testing together with informal feedback during a demo session has confirmed acceptance and learnability. However, in this paper we assess more closely the usability, performance and practical implications of this approach and thus focus on:

1. Description of design and functionality of the framework and its interface.

2. Head-to-head comparison of the OHW's performance against normal, non-enhanced operation.

3. Discussion of its suitability for different types of websites based on its implementation into popular sites.

4. Overall discussion of the OHW as a tool for onehanded website operation on touchscreen smartphones.

\section{PREVIOUS RESEARCH}

When designing thumb-friendly interfaces, Wobbrock et al. (Wobbrock et al., 2008) suggest supporting and evoking horizontal thumb movements as much as possible, as vertical movements were found to be overly challenging. On this basis they suggest a horizontal layout of interactive elements on the screen to accommodate the economic peculiarities of the thumb and improve usability. Katre (Katre, 2010) shows that a curved arrangement of elements on a touchscreen is perceived as comfortable and easy, as it supports a more natural circular motion of the thumb. An application of this is found in interfaces such as ArchMenu (Huot and Lecolinet, 2007) or ThumbMenu, where the user moves their thumb over an arch of elements placed in the bottom right corner of the screen.

Other researchers have explored the use of concentric menus such as the Wavelet menu (Francone et al., 2010) and SAM (Bonnet and Appert, 2011) to enhance thumb-based interaction, similar to the first generation Apple iPod. In the case of the Wavelet menu, research has shown that this approach with its consistent interaction model is easy to learn and efficient to use. Lü and $\mathrm{Li}$ ( $\mathrm{Lü}$ and $\mathrm{Li}, 2011$ ) present Gesture Avatar where the user can highlight a GUI element on screen to gain better control of it via an enlarged avatar. While this is an innovative way of improving one-handed device operation, it requires the user to draw their interface first, depends on a proprietary application and cannot be customised by the webmaster.

In terms of general adaptation of websites for mobile devices, one approach is web page segmenta- tion (Hattori et al., 2007; Gupta et al., 2007), using a proxy server to re-render the page into new logical units which are subsequently served to the device. A proprietary solution is the Read4Me browser (Yu and Miller, 2011) where the browser offers to optimise the page for mobile display via a proxy-server that then serves it to the user. Bandelloni et al. suggest a different system (Bandelloni et al., 2005) where the developer creates an abstract XML-based description of the layout and a proxy server renders the information for the respective access device. In addition to these techniques, various services, themes and frameworks exist to adapt websites for mobile devices and CSS3 media queries offer a flexible approach for display adaptation.

While existing approaches are all concerned with the display of a website on mobile devices, the OHW addresses a so-far neglected aspect: the specific support of one-handed operation of the web page. By implementing the OHW into a website, improvements are made to the operation - rather than the presentation - of the site, as this remains problematic even on well-adapted sites when operating it with one hand. Most importantly, the enhancement is done at runtime and on the client, can be fully configured by the webmaster and is dependent only on the browser itself and the user, who can choose to switch the enhancements on or off at any time.

\section{METHODOLOGY}

To verify the findings of previous researchers (Katre, 2010; Wobbrock et al., 2008) promoting a curved interface for thumb-based GUIs, we conducted a small user study with 7 participants $(3 \mathrm{~F}$, mean age 31.43 years, SD 4.65), all of who declared to be frequent users of touchscreen mobile devices. Participants were asked to swipe 10 times using their right and left thumb without looking at the device. They were instructed to swipe in a way that was most natural and comfortable to them, avoiding bending and stretching of the joints. Traces of these swipes were recorded on a hidden layer and saved. Stacking the resulting images on top of each other shows the curved movement created by a horizontal swipe, supporting the findings of previous researchers and informing our design of the interface (Fig. 1). To develop and verify our design, we iteratively tested paper prototypes with users to transform the wheel menu metaphor into a comprehensive website interface, supporting a more natural operation and minimal strain. Building on discussions with web developers, we made the OHW as non-intrusive and supportive as possible in the form of 


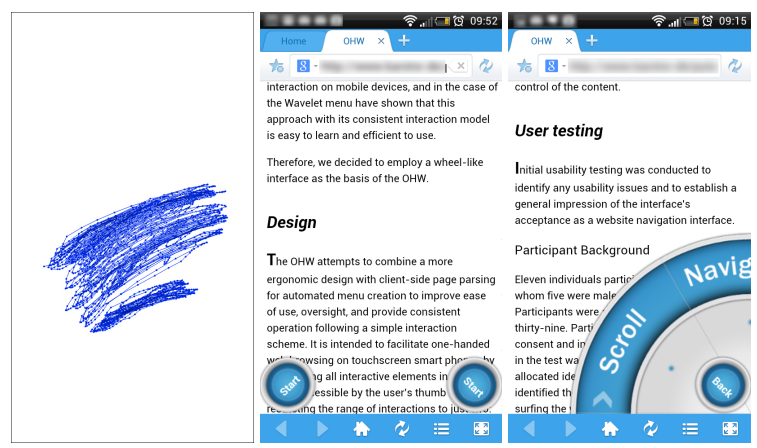

Figure 1: Visualisation of the swipe results (left), the OHW as it appears on start-up (middle) and launched (right).

an easily accessible, half-circle-shaped interface that can easily be added to a page by simply dropping the code into the website.

The OHW facilitates one-handed web browsing by assembling all interactive elements on request in a region easily accessible by the user's thumb and restricts the range of interactions to just two - swipe and tap. The layout of the page stays untouched and users can decide whether or not to use the interface at any time by switching it on or off (Fig. 1). Thus, the OHW is not an interface for mobile optimisation, which can be achieved using the techniques outlined above. Rather, the OHW's purpose is to enhance onehanded operation of a website regardless of its degree of adaptation, without spoiling the design. It augments the interaction model, not the display. To function, the OHW requires a browser with CSS3 support together with the jQuery JavaScript library - the most popular JavaScript library to date (Pingdom, 2010) - present on the website. Other than this, there are no minimum standards required and the OHW can be implemented into pages that already include libraries such as MooTools, for example. It has been trialled on a range of Android and iOS devices with HTML4 and HTML5 mark-up in Standards and Quirks mode.

The OHW interface consists of a variety of modules whose availability depends on the content of the website and the interface's configuration. Each module is represented as a wedge and together they form a wheel-type interface, either at the right-hand or lefthand bottom corner of the screen, depending upon the user's choice (Fig. 1). Only the modules that correspond to elements found on the page are loaded, but additional modules can be added at runtime by listening to updates of the Document Object Model (DOM).

To implement the OHW, the webmaster only needs to ensure that the jQuery JavaScript library is available on the website before linking to the OHW's code using a basic $<$ script $>$ tag. The webmaster

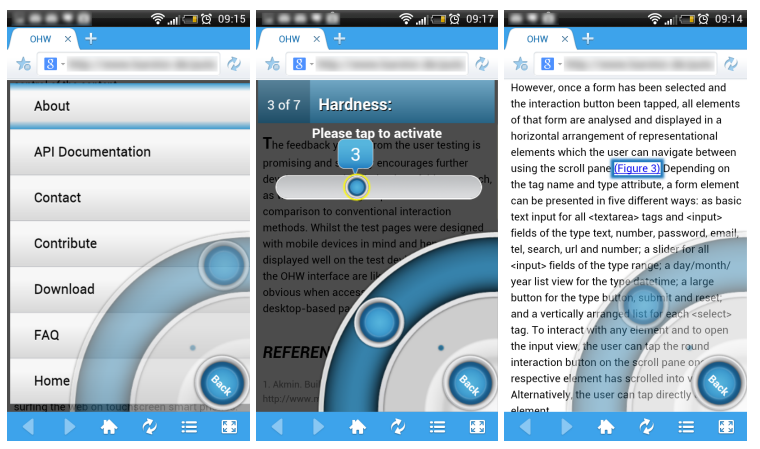

Figure 2: Basic list view of the site navigation (left), an augmented input field of the type range (middle) and the scroll functionality (right).

can optionally edit the configuration file, which is a JavaScript object, and adjust themes, selectors and custom functionality. As each and every aspect of the interface can be adjusted via CSS and HTML, the $\mathrm{OHW}$ can fit the look and content of a wide variety of websites. Once implemented, the code scans the website for certain tags from which to build the interface. By default these are basic HTML elements, such as $<$ nav $>$, $<$ video $>$, $<$ audio $>,<$ form $>,<$ h2 $>$, and $\langle\mathrm{a}\rangle$, and from these the standard names of the wedges are derived. This can easily be extended by using CSS selectors and custom wedges declared in the configuration file. The OHW contains several methods to cope with incorrect mark-up and can report any encountered problems to the webmaster.

The OHW's use is optional for the user and the interface can be hidden and brought back at any time. The interface is launched by tapping the Start button on either side of the screen to make it visible (Fig. 1). It can be spun by swiping over it to reveal all available functions. The Start button then becomes a Back button and can be used to either hide the interface completely or to go back one level. For example, if the user was standing and only had one hand free, they could tap the Start button and operate the site onehandedly with the help of the interface. As they sit down and free their other hand, they could hide the interface by tapping the Back button and continue to operate the website with both hands, without a change in design or presentation.

\section{FUNCTIONALITY}

The OHW offers improved presentation and onehanded operation for all interactive page elements. This can be achieved either by accessing them via the respective wedge in the wheel or by directly tapping them on the page. By default, the OHW uses basic 

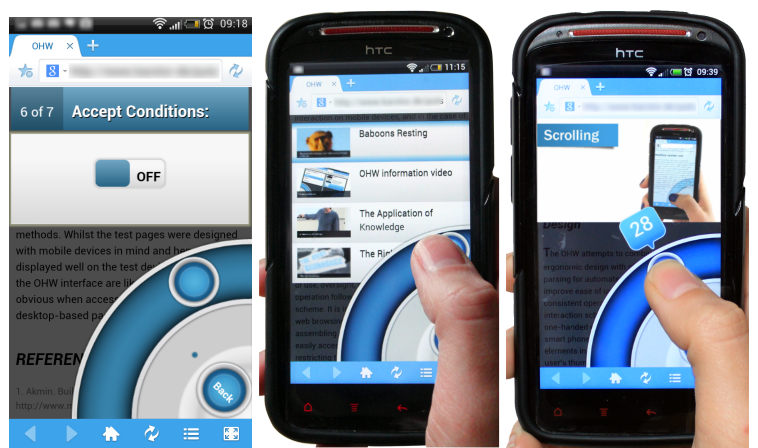

Figure 3: A checkbox input rendered by the OHW, where a tap on the OHW interface toggles the state (left), a user selecting a video from the media menu (middle) and video playback control (right).

list views (Fig. 2) that can hold images and text. In addition it offers on/off switches, sliders, buttons and a media player (Fig. 3, Fig. 4). These views are used in combination for the augmented presentations of otherwise hard to use elements and can be controlled by swiping and tapping. The $\mathrm{OHW}$ also provides scroll functionality similar to that of the Opera Mini browser (ASA, 2012). While scrolling, interactive elements closest to the current scroll position are outlined one at a time (Fig. 2) and can be activated by tapping the interface. Text input uses the system keyboard as initial tests showed that users found the OHW's own concentric keyboard hard to use. In addition to the functionality provided, the above can be easily extended by the webmaster by combining a CSS selector, one of the OHW's views and a custom function to suit their needs.

\section{INITIAL USER TESTING}

After iterative paper prototyping the interface was built and a pilot user test with 11 participants $(6 \mathrm{~F}$, all frequent smartphone users) was conducted to identify usability issues and to establish acceptance. Users were given a set of tasks one might perform on a page consisting of headlines, forms, videos, navigation and links, which they completed using the OHW without assistance. The page was designed to be deviceindependent using CSS3 media queries. All actions were recorded in video and audio, and feedback was given in a questionnaire on a five-point Likert scale. Feedback was predominantly positive and in response to the collected data we created an improved version of the interface for a usability study determining the efficiency and speed of the OHW in comparison to the normal operation (with one hand without the OHW) of a mobile-optimised website. During a demo ses-

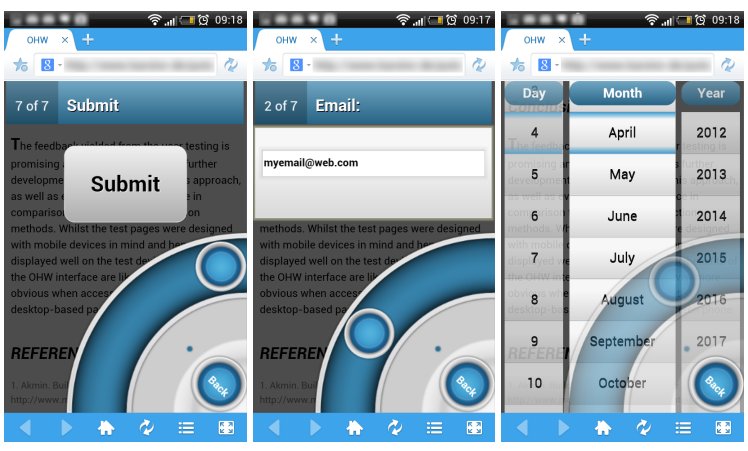

Figure 4: OHW representations of form elements: A button (left), a text input field (middle) and a date field. Users can swipe over the interface to navigate between elements in a form and tap to engage with the active element. Each element can be operated by performing swipe and tap actions on the interface.

sion at a conference (Seipp and Devlin, 2013) we presented the improved interface to visitors, implementing it on a range of popular websites to be experienced "hands-on". Informal feedback from users during these sessions was consistently positive, complimenting on the ease-of-use, usefulness and quality, thus supporting the validity of our approach to improve one-handed operation of websites on touchscreen mobile devices.

\section{USABILITY STUDY}

Altogether, 22 participants (7 F) aged 20 to 34 took part in the study, 19 of whom were final year undergraduate Computing students and the remainder were young professionals. All of them were righthanded, regular users of touchscreen mobile devices, such as phones and media players. The 19 Computing students were briefly introduced to the OHW during class and asked to do some self-directed exploring on a test page. On the day of the study, all users were given a 5-minute explanation of the usage of the OHW to ensure its operation was fully understood.

Using a within-subjects design, participants carried out the study one-handedly both with and without the use of the OHW. The study was counterbalanced by altering the mode in which the tasks were first performed. The first part of the usability study comprised 10 separate standard tasks a user might perform on a website and cover the whole spectrum of the OHW:

1. Finding a menu item in the navigation

2. Finding a video and forwarding it to a certain time

3. Finding a form and activating a checkbox

4. Finding another video and starting it 
5. Finding a form and filling in a date

6. Finding a link in the body text

7. Finding a form and filling in a range value

8. Finding a headline

9. Finding a form and pressing a button

10. Scrolling and clicking on a link

The study featured a website presenting the OHW. It was coded in HTML5 and CSS3 and contained a page navigation $(<$ nav $>)$, headlines $(<\mathrm{h} 1>,<\mathrm{h} 2>$, $<\mathrm{h} 3>$ ), a form with various elements of input types ("text", "range", "datetime", "submit", "checkbox"), paragraphs of text $(<\mathrm{p}>)$, three video files with poster images $(<$ video $>)$, links $(<\mathrm{a}>)$, images $(<\mathrm{img}>)$ as well as various $<$ div $>$ elements for the layout. These elements are very common and can be considered as representative for many websites. CSS3 media queries and relative measures were used to make the website's presentation device-independent. To conduct the study we used a HTC Sensation XE with Android 4.03 and the Maxthon Mobile Browser.

Tasks were performed directly on the website and were preceded by an instruction screen. Each task commenced from the top of the page. The number of interactions and time needed to accomplish the task were recorded using JavaScript. Recording began when users pressed $\mathrm{OK}$ on the instruction screen and stopped when a task was completed. For example, recording was only stopped once the target link was clicked or a certain value was entered into a field.

While the above is well-suited for determining efficiency on discrete tasks, it is less suitable for predicting the OHW's "real-life" performance on a page containing any number of these elements, where spatial proximity could affect performance. To address this we also measured the performance in 10 additional, consecutive tasks (1c to 10c), mimicking a set of coherent actions. After each part of this use case, recording was paused to show the instructions for the next part, but the current state of the website (scroll position, opened menus etc.) remained unchanged:

1c. Navigating to a headline in the text

2c. Scrolling and clicking on a link

3c. Finding a menu item in the navigation

4c. Finding a video and forwarding it to a certain time

5c. Navigating to another headline in the text

6c. Finding a link in the body text by scrolling

7c. Finding a form and entering a word into a text field

8c. Activating a checkbox in the same form

9c. Filling in a date in the same form

10c. Pressing a button in the same form

\section{RESULTS}

The data was evaluated using a Wilcoxon signed-rank test. Due to the varying, skewed results and small sample size we chose a series of non-parametric tests over the ANOVA. As tasks were not comparable in their results because of their different nature, they had to be treated as separate. Comparison of the one-handed task performance of users with the OHW against the normal, non-enhanced way draws a clear picture of the benefits the OHW offers to one-handed website operation. The effect of the OHW on efficiency can be derived from the median number of interactions required to perform a task (Table 1) as well as from the time needed to complete it (Table 2). Note: The values of the use case (shown in the tables as C) are based on the time and interactions needed to complete the whole use case, consisting of the 10 additional parts $1 \mathrm{c}$ to $10 \mathrm{c}$, forming one large task.

Table 1: Median interactions per task and the use case (C) w/out the OHW including $\mathrm{Z}$ and $p$ values as well as $\%$ of interactions $(\mathrm{I})$ needed with OHW $(\mathrm{Normal}=100 \%)$.

\begin{tabular}{|l|r|r|r|r|r|}
\hline Task & OHW & Normal & $\mathbf{Z}$ & $\boldsymbol{p}$ & \%I OHW \\
\hline $\mathbf{1}$ & 6 & 19 & 3.49 & $<.001$ & $32 \%$ \\
\hline $\mathbf{2}$ & 14 & 13.5 & 0.63 & .526 & $104 \%$ \\
\hline $\mathbf{3}$ & 14 & 22.5 & 2.93 & .003 & $62 \%$ \\
\hline $\mathbf{4}$ & 9 & 12.5 & 2.1 & .036 & $72 \%$ \\
\hline $\mathbf{5}$ & 26.5 & 27 & 0.15 & .884 & $98 \%$ \\
\hline $\mathbf{6}$ & 7.5 & 16 & 3.9 & $<.001$ & $47 \%$ \\
\hline $\mathbf{7}$ & 12 & 12.5 & 0.06 & .952 & $96 \%$ \\
\hline $\mathbf{8}$ & 14 & 19 & 1.97 & .049 & $74 \%$ \\
\hline $\mathbf{9}$ & 13.5 & 9.5 & 2.95 & .003 & $142 \%$ \\
\hline $\mathbf{1 0}$ & 51.5 & 26 & 3.98 & $<.001$ & $198 \%$ \\
\hline $\mathbf{C}$ & 104 & 127 & 2.18 & .029 & $82 \%$ \\
\hline
\end{tabular}

\subsection{Results: Number of Interactions Needed}

In 5 out of 10 tasks, the OHW allows users to complete the task with fewer interactions (Table 1). This is most visible in Tasks 1, 3 and 6 where the same task could be accomplished with only $32 \%, 62 \%$ and $47 \%$ of the interactions required without the interface. This highlights the OHW's enhancement of tasks such as finding an item in the navigation, operating a checkbox and retrieving a link from the body text. Other tasks that took fewer interactions to perform with the OHW than without were locating a video (Task 4, $72 \%$ ) and finding a headline (Task 8, 74\%). However, the results also show areas where more interac- 
tions are required with the OHW than without. This includes finding and pressing a submit button (Task 9, 142\%) and lengthy scrolling to find a link (Task 10, $198 \%)$.

\subsection{Results: Amount of Time Needed}

Evaluating OHW performance based on the actual time needed to complete a task draws an even clearer picture of the OHW's effectiveness (Table 2). Using the OHW in all tasks but one is significantly faster than performing the same tasks without the OHW. The most striking differences can be observed in Task 1 (33\% of the time needed), Task $3(47 \%)$, Task 4 $(36 \%)$, Task $6(33 \%)$ and Task $8(46 \%)$. However, scrolling through the document (Task 10) takes more time with the OHW (147\% of time needed).

Table 2: Median time (T) in seconds needed per task (1 to $10)$ and the use case (C) w/out the OHW including $\mathrm{Z}$ and $p$ values as well as \% of time needed with the OHW (Normal $=100 \%$ ).

\begin{tabular}{|l|r|r|r|r|r|}
\hline Task & OHW & Normal & $\mathbf{Z}$ & $\boldsymbol{p}$ & \% T OHW \\
\hline $\mathbf{1}$ & 11.40 & 34.30 & 4.11 & $<.001$ & $33 \%$ \\
\hline $\mathbf{2}$ & 25.90 & 45.10 & 4.07 & $<.001$ & $57 \%$ \\
\hline $\mathbf{3}$ & 20.50 & 43.90 & 4.11 & $<.001$ & $47 \%$ \\
\hline $\mathbf{4}$ & 10.20 & 28.60 & 4.11 & $<.001$ & $36 \%$ \\
\hline $\mathbf{5}$ & 33.90 & 46.60 & 3.98 & $<.001$ & $73 \%$ \\
\hline $\mathbf{6}$ & 9.50 & 29.10 & 4.11 & $<.001$ & $33 \%$ \\
\hline $\mathbf{7}$ & 18.80 & 26.30 & 3.85 & $<.001$ & $72 \%$ \\
\hline $\mathbf{8}$ & 14.80 & 31.90 & 4.07 & $<.001$ & $46 \%$ \\
\hline $\mathbf{9}$ & 15.50 & 22.10 & 3.17 & .002 & $70 \%$ \\
\hline $\mathbf{1 0}$ & 65.40 & 44.50 & 3.56 & $<.001$ & $147 \%$ \\
\hline $\mathbf{C}$ & 153.20 & 255.10 & 4.11 & $<.001$ & $60 \%$ \\
\hline
\end{tabular}

\subsection{Results of the Use Case}

The results of the use case show that in a real-life application the impact of the OHW on efficiency is significant, as overall it took participants only $60 \%$ of the time and $82 \%$ of the interactions when using the OHW as opposed to operating the website normally (Table 2 and Table 1).

\subsection{Implementation into Popular Websites}

To determine the OHW's versatility and suitability for different types of websites, we implemented it on several popular websites via a proxy script that injected the OHW code into the loaded page with the following results:

\subsubsection{Wikipedia}

(Wikipedia, 2013) The mobile article view divides the content into sections which can be expanded, each headed by an $\langle\mathrm{h} 2\rangle$. Out of the box the OHW was useful for quick access to the navigation, links, search and scrolling, but not as useful for jumping to a headline, as the user would still have to tap the element on the page to expand it. Overall the interface was quick to load and very responsive, but the number of links on the page shown in the OHW Links menu totalled 538. This resulted in a very large list with jerky scrolling despite the use of hardware-accelerated CSS transitions.

\subsubsection{BBC News}

(BBC, 2013) Standard configuration offered quick access to the page navigation and search form, but the headlines menu combined stories from all sections, making it hard for the user to discern where a story belonged. This indicates that the content to be made accessible by the OHW needs to be defined by the webmaster when configuring the $\mathrm{OHW}$ using custom wedges and selectors. Start-up was quick and operation was very smooth. The Headlines menu held 32 items, the Links menu 15 and the Navigation menu 35 items. Implementing the $\mathrm{OHW}$ on the desktop version of the site, however, exposes a weakness of this client-side approach. The desktop contains various JavaScript-based fading animations which heavily impact performance on mobile devices. With these animations, the otherwise smooth operation was occasionally interrupted as the $\mathrm{OHW}$ has to share the processing resources with other page elements.

\subsubsection{W3C}

(W3C, 2013) The OHW performed very smoothly with 20 items in the Links menu, 10 items in the Navigation menu and 22 items in the Headlines menu. As stated previously, the labels of the wedges could use simple customisation by the webmaster to reflect the content of the website.

\subsubsection{Google}

(Google, 2013) The OHW was quick to load and very responsive with 11 items in the Navigation menu, 10 items in the Results menu (Headlines), and 11 items in the Links menu. Some customisation is required to better match the content. 


\subsubsection{WordPress Blog}

(WordPress, 2013) The OHW performed smoothly with 6 items in the Navigation menu, 16 items in the Links menu and 12 items in the Posts menu (Headlines), but again the wedges could be renamed to represent the content meaningfully.

\subsubsection{YouTube}

(YouTube, 2013) Smooth performance with a basic configuration showing 4 items in the Navigation menu, 9 items in the Videos (Headlines) menu and 6 items in the Links menu. Unfortunately, videos could not be played back within the OHW as these were served as $3 \mathrm{gp}$ files. The OHW can only play back files which are natively supported by the browser.

\subsubsection{Flickr}

We were unable to receive the mobile version of the site (Flickr, 2013) using our proxy script, despite manually altering the header information of the request to mimic a mobile device. Attempts to mirror parts of the site locally did not allow sufficiently accurate reconstruction of the mobile view either.

\subsection{General Performance}

Performance of the OHW (Table 3) was tested in the standard browser with varying amounts of content on an HTC Sensation XE with Android 4.03 and an iPhone 3GS with iOS 6.1. First, we measured the start-up time of the interface on each device on the websites discussed in the previous section. Then we measured the time it took each device to create a list view with varying amounts of items after tapping a wedge in the wheel. For this we chose the WordPress blog (WordPress, 2013) as a base and injected additional elements into the DOM when fetching the page using the proxy script. All measurements were performed three times on each device with a cleared browser cache.

\section{DISCUSSION}

First we discuss usability and efficiency from a user perspective. Next we discuss the performance of the framework to determine the boundaries in which it can be deployed.
Table 3: Mean time in seconds needed by the HTC Sensation XE (S. XE) and iPhone 3GS (3GS) to create a list view (Fig. 1, right) after tapping a wedge in the wheel. Second part shows mean start-up time (SU) when implemented on a website.

\begin{tabular}{|l|l|l|}
\hline Task & S. XE & 3GS \\
\hline List view, 30 items & 1.1 & 0.6 \\
\hline List view, 60 items & 1.5 & 0.7 \\
\hline List view, 120 items & 1.9 & 0.7 \\
\hline List view, 480 items & 2.4 & 0.6 \\
\hline SU Wikipedia & 1.4 & 0.9 \\
\hline SU BBC News & 2.3 & 1.4 \\
\hline SU W3C & 0.3 & 0.8 \\
\hline SU Google & 0.3 & 1.0 \\
\hline SU WordPress & 1.5 & 1.4 \\
\hline SU YouTube & 0.9 & 0.9 \\
\hline
\end{tabular}

\subsection{Usability and Efficiency}

When operating a website with only one hand, the OHW presents a clear advantage over the normal, non-enhanced mode of operation. In the majority of cases, using the OHW requires less interaction to complete a task and in $90 \%$ of the examined cases, a task is completed significantly faster when using the OHW. However, the results also highlight a weak point of the OHW. When the user has to scroll a large section of the page, the performance of the OHW is significantly weaker than the normal mode of operation ( $147 \%$ of time needed). It shows that scrolling with one hand is already very efficient and that the OHW's approach cannot compete with the existing solution, but needs improvement. This has since been addressed by combining native scrolling and $\mathrm{OHW}$ scrolling so that the user can scroll the website as usual, but can make more precise selections by moving their thumb over the interface at the same time.

\subsection{Versatility}

Implementation into different types of websites highlights the pros and cons of our approach. Customisation of the OHW is easy: the webmaster can quickly adapt the text of the wedges to reflect website content using the supplied templates. Custom functionality is achieved by using the OHW's plugin model to accommodate a website's own set of interactions, such as the accordion-like blocks on Wikipedia (Wikipedia, 2013) with custom callback functions. Thus configured, the OHW is suited to pages with categorised 


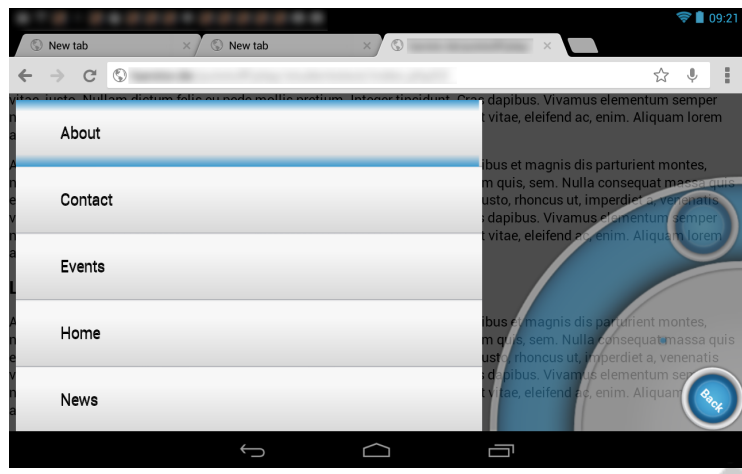

Figure 5: The OHW on a Nexus 7 in horizontal orientation.

text and images that stretch over many screens and would otherwise need scrolling to access, as found on news websites, wikis, forums, blogs and search engines. Benefits for all types of websites include quick access and operation of forms, navigation, and control of audio or video items if supported natively by the browser.

The OHW performs well on mobile-adapted pages if the content per menu is not excessive. Operation is smooth even with 120 items to be displayed and scrolled. Beyond that the list scrolling performance decreases on the HTC Sensation XE with the amount of data to be presented, whereas it stays the same on the iPhone 3GS with up to 480 list items. While this decrease is likely to only happen in rare cases on mobile websites - as observed in our Wikipedia test - it is more likely to occur on desktop-oriented pages due to the larger page load and other resource-depleting processes. Therefore the webmaster has to be considerate when implementing the OHW: a site loaded with badly coded animations that already struggles being displayed on a mobile device will not necessarily be improved by the OHW. This highlights the main problem of using an integrated client-side approach: the interface has to share the resources with the content of the website, which can directly influence performance. Luckily, this is in the hands of the webmaster implementing the OHW and thus straightforward to address. However, it also shows that the OHW is not a magical one-size-fits-all solution for making any website easier to interact with when operating the device with one hand. What it does, though, is significantly improve operation and efficiency on already mobile-adapted websites (Table 2) together with a short start-up time and high responsiveness (Table 3).

\section{CONCLUSION}

Our research shows that applying the wheel-menu metaphor as the basis for thumb-based website interaction and offering a curved input control based on solely swipe and tap for all interactive elements clearly improves one-handed website operation and allows users to complete their goals more quickly and comfortably as they do not have to loosen their grip on the device when trying to reach elements outside the arc of their thumb. Given the demand for a simple, one-handed way to access websites on a mobile device (Karlson and Bederson, 2006), the OHW is a practical and highly effective solution from both a web developer and end-user perspective, if the technical requirements are met. The $\mathrm{OHW}$ promotes a free and inclusive way of improving user experience on the mobile web for modern touchscreen smartphone users and supports openness and flexibility. The use of standard web technologies allows it to easily adapt to new challenges and ensures its longevity and ease-of-use for the webmaster. Future work will address performance optimisations for the operation of large lists and the development of a SVG and core JavaScript implementation. We plan to evaluate the OHW's applicability as an interface for HTML5based smartphone apps and the development of an extended plugin model to allow more advanced custom functionality. As it stands, the $\mathrm{OHW}$ is a promising approach for enhancing one-handed web browsing on a wide range of mobile touchscreen devices (Fig. 5).

\section{REFERENCES}

Akmin (2012). Build your own mobile website ... in minutes. http://www.mobisitegalore.com/index.html.

ASA, O. S. (2007). Making small devices look great. http://dev.opera.com/articles/view/makingsmall-devices-look-great.

ASA, O. S. (2012). http://www.opera.com/mobile/specs.

Bandelloni, R., Mori, G., and Paternò, F. (2005). Dynamic generation of web migratory interfaces. In Proc. Mobile HCI 2005, pages 83-90, New York, NY, USA. ACM.

BBC (2013). BBC news. http://m.bbc.co.uk/news.

Bonnet, D. and Appert, C. (2011). Sam: the swiss army menu. In Proc. IHM 2011, pages 5:1-5:4, New York, NY, USA. ACM.

Envato (2013). Signum mobile - html5 \& css3 and iwebapp. http://themeforest.net/item/signum-mobilehtml5-css3-and-iwebapp/1614712.

Flickr (2013). Flickr. http://m.flickr.com.

Francone, J., Bailly, G., Lecolinet, E., Mandran, N., and Nigay, L. (2010). Wavelet menus on handheld devices: 
stacking metaphor for novice mode and eyes-free selection for expert mode. In Proc. AVI 2010, AVI '10, pages 173-180, New York, NY, USA. ACM.

Google (2013). Google search results. https://www.google.co.uk/search?q=something.

Gupta, A., Kumar, A., Mayank, Tripathi, V. N., and Tapaswi, S. (2007). Mobile web: web manipulation for small displays using multi-level hierarchy page segmentation. In Proc. MCO7 4th Mobility Conference, pages 599-606. ACM.

Hattori, G., Hoashi, K., Matsumoto, K., and Sugaya, F. (2007). Robust web page segmentation for mobile terminal using content-distances and page layout information. In Proc. WWW 2007, pages 361-370. ACM.

Huot, S. and Lecolinet, E. (2007). Archmenu et thumbmenu: contrôler son dispositif mobile "sur le pouce". In Proc. IHM 2007, pages 107-110, New York, NY, USA. ACM.

Karlson, A. K. and Bederson, B. B. (2006). Studies in onehanded mobile design: Habit, desire and agility. Technical report, Computer Science Dept., Uni. of Maryland.

Katre, D. (2010). One-handed thumb use on smart phones by semi-literate and illiterate users in india. In HWID. Usability in Social, Cultural and Organizational Contexts, volume 316, pages 189-208. Springer Boston.

Lü, H. and Li, Y. (2011). Gesture avatar: a technique for operating mobile user interfaces using gestures. In Proc. CHI 2011, pages 207-216. ACM.

Mobotap (2012). Dolphin browser. http://dolphinbrowser.com/.

Pingdom (2010). jQuerys triumphant march to success. http://royal.pingdom.com/2010/03/26/jquerytriumphant-march-to-success/.

Seipp, K. and Devlin, K. (2013). Enhancing one-handed website operation on touchscreen mobile phones. In CHI '13 Extended Abstracts on Human Factors in Computing Systems, CHI EA '13, pages 3123-3126, New York, NY, USA. ACM.

W3C (2008). Mobile web best practices 1.0. http://www.w3.org/TR/mobile-bp/.

W3C (2013). W3C. http://www.w3.org.

Wikipedia (2013). Deusdedit of Canterbury. http://en.m.wikipedia.org/wiki/Deusdedit_of_Canterbury.

Wobbrock, J. O., Myers, B. A., and Aung, H. H. (2008). The performance of hand postures in front- and backof-device interaction for mobile computing. Int. J. Hum.-Comput. Stud., 66(12):857-875.

WordPress (2013). Just another wordpress weblog. http://en.blog.wordpress.com/.

YouTube (2013). Youtube mobile. http://www.youtube.com/results?client=mvgoogle \&q=sublime.

Yu, C.-H. and Miller, R. C. (2011). Enhancing mobile browsing and reading. In Ext. Abstracts Proc. CHI 2011, pages 1783-1788. ACM. 